\title{
Lusioersily
}

\section{Fairness-Oriented Resource Allocation for Energy Efficiency Optimization in Uplink OFDMA Networks}

Sokun, H. U., Mohamed, E. B., Gohary, R., \& Yanikomeroglu, H. (2018). Fairness-Oriented Resource Allocation for Energy Efficiency Optimization in Uplink OFDMA Networks. 2018 IEEE Wireless Communications and Networking Conference (WCNC). https://doi.org/10.1109/WCNC.2018.8377327

Link to publication record in Ulster University Research Portal

Published in:

2018 IEEE Wireless Communications and Networking Conference (WCNC)

Publication Status:

Published (in print/issue): 11/06/2018

DOI:

10.1109/WCNC.2018.8377327

\section{Document Version}

Author Accepted version

\section{General rights}

Copyright for the publications made accessible via Ulster University's Research Portal is retained by the author(s) and / or other copyright owners and it is a condition of accessing these publications that users recognise and abide by the legal requirements associated with these rights.

\section{Take down policy}

The Research Portal is Ulster University's institutional repository that provides access to Ulster's research outputs. Every effort has been made to ensure that content in the Research Portal does not infringe any person's rights, or applicable UK laws. If you discover content in the Research Portal that you believe breaches copyright or violates any law, please contact pure-support@ulster.ac.uk. 


\title{
Fairness-Oriented Resource Allocation for Energy Efficiency Optimization in Uplink OFDMA Networks
}

\author{
Hamza Umit Sokun, Ebrahim Bedeer, Ramy H. Gohary, and Halim Yanikomeroglu
}

\begin{abstract}
Due to the battery-limited nature of mobile devices, improving energy efficiency (EE) of individual users and ensuring EE fairness among those users are one of the key design issues in uplink transmission of cellular networks. In this paper, we consider the joint optimization of discrete power and resource blocks allocations to maximize the minimum EE among users subject to individual power budget constraints. The optimization problem is combinatorial. Thus, we propose an efficient algorithm, based on semidefinite relaxation with Gaussian randomization, to solve the resultant non-convex problem in polynomial time complexity. The numerical results show how well the proposed algorithm performs against the optimal one and indicate the impact of discrete power levels on the fairness-oriented EE optimization.

Index Terms-Energy efficiency, max-min fairness, OFDMA, convex optimization, semidefinite relaxation, randomization.
\end{abstract}

\section{INTRODUCTION}

Today's mobile devices become an irreplaceable part of our life with a wide range of new emerging applications and services, e.g., GPS navigation, e-healthcare, and mobile video conference. However, these advance applications and services require not only higher data rates [1], but also higher energy consumption. Such a rise in energy consumption adversely affects the battery life of mobile devices that are typically powered by pre-charged batteries. To prolong the battery life of the devices, for all users, it is important to have the highest energy efficiency (EE) possible. However, this will lead to a problem of EE fairness (balancing) among users, particularly in uplink transmissions, as each individual will want to maximize their own benefits. Hence, for current and next-generation wireless networks, not only improving users' EE, but also ensuring EE fairness among users are critically important design requirements.

Generally speaking, for improving the users' EE, their data rate needs to be increased, while at the same time, their energy consumption needs to be decreased. To achieve these objectives, resource allocation is an effective tool [2]. More specifically, optimizing the way how the available resource blocks (RBs) in the network are allocated among the users, and the way how the users allocate their available power across those RBs can substantially enhance the users' EE [3]. For further enhancement in the users' $\mathrm{EE}$, the interaction between these network functionalities should be considered.

\section{A. Prior Work and Contribution}

Despite the rich literature on the overall EE optimization in the downlink of an orthogonal frequency division multiple access (OFDMA)-based systems, fairnessoriented individual EE optimization in the uplink of OFDMA-based systems is not thoroughly investigated from resource allocation perspective. There are only a limited number of works in the literature studied this problem (see, e.g., [4]-[7]). In [4], the power optimization problem with fixed RB allocation is considered for uplink transmission in OFDMA systems to maximize the minimum EE of all users. Similarly, in [5], the power optimization problem is discussed for uplink transmission in spectrum-sharing networks to achieve the proportional, harmonic, and max-min fair EE. On the other hand, the problem of the joint optimization of subcarrier and power allocation is addressed in [6] to achieve the max-min EE fairness in the uplink transmission of OFDMA networks. Lastly, in [7], the problem of the joint optimization of subcarrier and and power allocation is investigated to attain the max-min EE fairness in the uplink transmission of amplify-and-forward cooperative OFDMA networks. However, it is important to mention that the resource allocation techniques proposed in [4][7] are based on continuous power allocation, rather than allocation of discrete power levels. Hence, implementing such resource allocation techniques in practical networks supporting discrete power levels, e.g., LTE networks, can be problematic.

In this work, we propose a joint resource allocation technique to maximize the minimum $\mathrm{EE}$ of all users in OFDMA networks, while taking practical implementation constraints into account, i.e., including discrete power levels and per-user power budget. In particular, we consider the joint design of RB and discrete power allocations, and formulate the joint design as a maxmin EE optimization problem. Restricting the power levels for being discrete has several advantages, such as simplifying the hardware designs, and enabling a low 
cost implementation, however, this usually leads to nonconvex formulations. To overcome this difficulty, we consider the semidefinite relaxation (SDR)-based Gaussian randomization technique that invokes a relaxation on the rank of the matrix-value optimization variable to obtain near-optimal results in polynomial time.

\section{Problem Statement And Preliminaries}

This section describes the network model under consideration, and discusses the optimization problem formulation.

\section{A. System Description}

We consider an uplink OFDMA network which consists of a base station (BS), $M$ users, $J$ RBs and $L$ discrete power levels. Let $\mathcal{M}=\{1, \ldots, M\}$ denote the set of users, $\mathcal{J}=\{1, \ldots, J\}$ denote the set of RBs and $\mathcal{P}=\left\{p^{1}, \ldots, p^{\ell}, \ldots, p^{L}\right\}$ denote the set of power levels, where $L=|\mathcal{P}|$ is the cardinality of $\mathcal{P}$. Let $h_{m}^{j}$ represent the channel gain for the $m$-th user on the $j$-th RB. Then, the maximum achievable rate for $m$-th user on the $j$-th $\mathrm{RB}$ when $\ell$-th power level is used can be expressed as

$$
r_{m}^{j \ell}=B \log _{2}\left(1+\frac{p^{\ell}\left|g_{m}^{j}\right|^{2}}{B N_{0}}\right),
$$

where each RB has a bandwidth of $B$ and $N_{0}$ is the power spectral density of additive white Gaussian noise. Additionally, let binary variable $x_{m}^{j \ell}$ be an indicator variable that indicates whether the $m$-th user is associated with the BS on the $j$-th RB using the $\ell$-th power level or not. Using the indicator variables, the overall transmission rate for the $m$-th user can be cast as $\sum_{j \in \mathcal{J}} \sum_{\ell \in \mathcal{P}} r_{m}^{j \ell} x_{m}^{j \ell}$ Moreover, to avoid interference among RBs, each RB is allocated to a single user at most, so that total usage of the $j$-th RB over the network should satisfy

$$
\sum_{m \in \mathcal{M}} \sum_{\ell \in \mathcal{P}} x_{m}^{j \ell} \leq 1, \quad \forall j \in \mathcal{J}
$$

Energy Efficiency Metric: The power consumption for the $m$-th user is composed of two components. The first is the total transmit power consumption for $m$-th user to the BS, which can be expressed as $\sum_{j \in \mathcal{J}} \sum_{\ell \in \mathcal{P}} p^{\ell} x_{m}^{j \ell}$ Noting that in practice, this consumption is limited by a threshold, $P_{m}^{\max }$, so that it should satisfy

$$
\sum_{j \in \mathcal{J}} \sum_{\ell \in \mathcal{P}} p^{\ell} x_{m}^{j \ell} \leq P_{m}^{\max }, \quad \forall m \in \mathcal{M} .
$$

The second is circuitry power consumption for the $m$-th user to the BS, $P_{m, \mathrm{C}}$. As a result, the overall power consumption at the $m$-th user

$P_{m, \mathrm{~T}}=P_{m, \mathrm{C}}+\frac{1}{\rho} \sum_{j \in \mathcal{J}} \sum_{\ell \in \mathcal{P}} p^{\ell} x_{m}^{j \ell}, \forall m \in \mathcal{M}$,

where $\rho$ is the power amplifier efficiency constant.
The EE for each user is defined as the ratio between the total data rate and the total consumed power:

$$
\mathcal{E}_{m, \mathrm{EE}}=\frac{\sum_{j \in \mathcal{J}} \sum_{\ell \in \mathcal{P}} r_{m}^{j \ell} x_{m}^{j \ell}}{P_{m, \mathrm{~T}}} \text { bits/Joule, } \forall m \in \mathcal{M} \text {. }
$$

Fairness Metric: For measuring the EE fairness between the users with the battery operated devices, we adopt a (non-numeric) qualitative metric, i.e., maxmin fairness index. When the network reaches max-min EE fairness, any user's EE cannot be increased without decreasing another user's EE.

\section{B. Problem Formulation for Fairness-Oriented Energy Efficiency Optimization}

We consider RB and power allocations jointly to obtain a max-min energy-efficient resource allocation strategy in the uplink OFDMA networks. In particular, this strategy aims to maximize the minimum EE among all users while guaranteeing the total transmit power per user is not exceeded. We formulate the max-min optimization problem as follows:

$$
\max _{\left\{x_{m}^{j \ell}\right\}} \min _{m \in \mathcal{M}} \mathcal{E}_{m, \mathrm{EE}}=\frac{\sum_{j \in \mathcal{J}} \sum_{\ell \in \mathcal{P}} r_{m}^{j \ell} x_{m}^{j \ell}}{\frac{1}{\rho} \sum_{j \in \mathcal{J}} \sum_{\ell \in \mathcal{P}} p^{\ell} x_{m}^{j \ell}+P_{m, \mathrm{C}}},
$$

$$
\begin{aligned}
\text { subject to } & \sum_{m \in \mathcal{M}} \sum_{\ell \in \mathcal{P}} x_{m}^{j \ell} \leq 1, \quad \forall j \in \mathcal{J}, \\
& \sum_{j \in \mathcal{J}} \sum_{\ell \in \mathcal{P}} p^{\ell} x_{m}^{j \ell} \leq P_{m}^{\max }, \quad \forall m \in \mathcal{M}, \\
& x_{m}^{j \ell} \in\{0,1\}, \forall m \in \mathcal{M}, \forall j \in \mathcal{J}, \forall \ell \in \mathcal{P} .
\end{aligned}
$$

Noting that the objective is fractional, and the constraint in (6d) is non-convex. Hence, the optimization problem in (6) is a fractional integer non-linear programming problem. Finding out the optimal solution for such a non-convex problem requires exponential complexity and is computationally prohibitive. Hence, we propose a twosteps polynomial-time algorithm that based on the SDR technique with Gaussian randomization [8] to provide an approximate solution with high accuracy. In the next section, we will elaborate on the proposed algorithm.

\section{PROposed SOLUTION}

The idea behind the SDR technique ${ }^{1}$ is that the original non-convex problem is first reformulated in a higher dimension, and next, the non-convex constraints are relaxed. The resulting convex problem is lastly used to approximate the original one. To facilitate formulating

\footnotetext{
${ }^{1}$ A somewhat similar technique is used in [9] to address a different optimization problem in a different context. More specifically, in [9], maximizing the network-side overall $\mathrm{EE}$ is discussed in downlink of OFDMA-based systems, whereas herein the design objective is to improve minimum individual $\mathrm{EE}$ as much as possible in uplink of OFDMA-based systems.
} 
the optimization problem in a higher dimension, we define the following five 3-dimensional tensors: $\boldsymbol{A}_{j}$, $\boldsymbol{B}_{m}, \boldsymbol{G}_{m}$, and $\boldsymbol{X}$. These tensors are in the form of a $J \times M L$ block-partitioned matrix with $1 \times M$ blocks, each with $J \times L$ entries. The tensor $\boldsymbol{X}$ can be expressed as $\boldsymbol{X}=\left[\begin{array}{lll}\boldsymbol{X}_{1} & \ldots & \boldsymbol{X}_{M}\end{array}\right]$, where, for all $m=1, \ldots, M$, $\boldsymbol{X}_{m}$ is given as $\boldsymbol{X}_{m}=\left[\begin{array}{ccc}x_{m}^{11} & \ldots & x_{m}^{1 \ell} \\ \vdots & \ddots & \vdots \\ x_{m}^{J 1} & \ldots & x_{m}^{J L}\end{array}\right]$. The tensor $\boldsymbol{A}_{j}$ can be given as $\boldsymbol{A}_{j}=\left[\begin{array}{lll}\boldsymbol{A}_{j_{1}} & \ldots & \boldsymbol{A}_{j_{M}}\end{array}\right], \quad j=$ $1, \ldots, J$, where for all $m=1, \ldots, M, \boldsymbol{A}_{j_{m}}=$ $\boldsymbol{e}_{j} \mathbf{1}_{L}^{T}$, where $\boldsymbol{e}_{j}$ is the $j$-th column of the $J \times J$ identity matrix $\boldsymbol{I}_{J}$. The tensor $\boldsymbol{B}_{m}$ can be written as $\boldsymbol{B}_{m}=\left[\begin{array}{lllll}\boldsymbol{B}_{m_{1}} & \ldots & \boldsymbol{B}_{m_{\hat{m}}} & \ldots & \boldsymbol{B}_{m_{M}}\end{array}\right], \quad m=$ $1, \ldots, M$, where for $i=1, \ldots, M, \boldsymbol{B}_{m_{i}}=\mathbf{0}_{J \times L}$ when $i \neq \hat{m}$, and, when $i=\hat{m}, \boldsymbol{B}_{m_{\hat{m}}}=$ $\left[\begin{array}{ccc}r_{\hat{m}}^{11} & \cdots & r_{\hat{m}}^{1 \ell} \\ \vdots & \ddots & \vdots \\ r_{\hat{m}}^{J 1} & \ldots & r_{\hat{m}}^{J L}\end{array}\right]$. The tensor $\boldsymbol{G}_{m}$ can be expressed as $\boldsymbol{G}_{m}=\left[\begin{array}{lllll}\boldsymbol{G}_{m_{1}} & \ldots & \boldsymbol{G}_{m_{\hat{m}}} & \ldots & \boldsymbol{G}_{m_{M}}\end{array}\right], \quad m=$ $1, \ldots, M$, where for $i=1, \ldots, M, \boldsymbol{G}_{m_{i}}=\mathbf{0}_{J \times L}$ when $i \neq \hat{m}$, and, when $i=\hat{m}, \boldsymbol{G}_{m_{\hat{m}}}=\left[\begin{array}{ccc}p^{1} & \cdots & p^{L} \\ \vdots & \ddots & \vdots \\ p^{1} & \cdots & p^{L}\end{array}\right]$. Subsequently, we obtain the following vectors: $\boldsymbol{x} \triangleq$ $\operatorname{vec}\left(\boldsymbol{X}^{T}\right), \boldsymbol{a}_{j} \triangleq \operatorname{vec}\left(\boldsymbol{A}_{j}^{T}\right), \boldsymbol{b}_{m} \triangleq \operatorname{vec}\left(\boldsymbol{B}_{m}^{T}\right)$, and $\boldsymbol{g}_{m} \triangleq$ $\operatorname{vec}\left(\boldsymbol{G}_{m}^{T}\right)$. Additionally, we introduce the vector $\boldsymbol{x} \in$ $\{0,1\}^{M J L}$. Using the defined vectors, the optimization problem can be cast as

$$
\begin{array}{cl}
\max _{\boldsymbol{x}} \min _{m \in \mathcal{M}} & \frac{\boldsymbol{b}_{m}^{T} \boldsymbol{x}}{\frac{1}{\rho} \boldsymbol{g}_{m}^{T} \boldsymbol{x}+P_{m, \mathrm{C}}}, \\
\text { subject to } & \boldsymbol{a}_{j}^{T} \boldsymbol{x} \leq 1, \quad \forall j \in \mathcal{J}, \\
& \boldsymbol{g}_{m}^{T} \boldsymbol{x} \leq P_{m}^{\max }, \quad \forall m \in \mathcal{M}, \\
& \boldsymbol{x} \in\{0,1\}^{M J L} .
\end{array}
$$

Using the vector $\boldsymbol{y} \in\{-1,1\}^{M J L}$, where $\boldsymbol{y}=2 \boldsymbol{x}-\mathbf{1}$, we reformulate the problem in (6) as

$$
\begin{aligned}
& \max _{\boldsymbol{y}} \min _{m \in \mathcal{M}} \frac{\boldsymbol{b}_{m}^{T}(\boldsymbol{y}+\mathbf{1})}{\frac{1}{\rho} \boldsymbol{g}_{m}^{T}(\boldsymbol{y}+\mathbf{1})+2 P_{m, \mathrm{C}}}, \\
& \text { subject to } \quad \frac{1}{2} \boldsymbol{a}_{j}^{T}(\boldsymbol{y}+\mathbf{1}) \leq 1, \quad \forall j \in \mathcal{J}, \\
& \frac{1}{2} \boldsymbol{g}_{m}^{T}(\boldsymbol{y}+\mathbf{1}) \leq P_{m}^{\max }, \quad \forall m \in \mathcal{M}, \\
& \boldsymbol{y} \in\{-1,1\}^{M J L} .
\end{aligned}
$$

It is worth to mention that the constraints in (8b) and (8c) are linear. However, since the objective (8a) and the constraint (8d) are non-convex, the problem in (8) is still non-convex.

The next step for applying the SDR technique is to formulate the optimization problem of (8) in a higher dimension, i.e., the optimization variables are replaced with symmetric positive semidefinite (PSD) matrices [8]. Before proceeding with the next step, we also define the following vectors in $\mathbb{R}^{M J L+1}, \hat{\boldsymbol{g}}_{m} \triangleq$ $\left[\frac{1}{\rho} \boldsymbol{g}_{m}^{T} \frac{1}{\rho} \boldsymbol{g}_{m}^{T} \mathbf{1}+2 P_{m, \mathrm{C}}\right]^{T}, \hat{\boldsymbol{a}}_{j} \triangleq\left[\boldsymbol{a}_{j}^{T} \boldsymbol{a}_{j}^{T} \mathbf{1}\right]^{T}, j=1, \ldots, J$, $\hat{\boldsymbol{b}}_{m} \triangleq\left[\begin{array}{ll}\boldsymbol{b}_{m}^{T} & \boldsymbol{b}_{m}^{T} \mathbf{1}\end{array}\right]^{T}, m=1, \ldots, M, \hat{\mathbf{1}} \triangleq\left[\begin{array}{ll}\mathbf{1}^{T} & \mathbf{1}^{T} \mathbf{1}\end{array}\right]^{T}$, $\hat{\boldsymbol{y}} \triangleq\left[\begin{array}{ll}\boldsymbol{y}^{T} & 1\end{array}\right]^{T}$ and $\hat{\boldsymbol{f}} \triangleq\left[\begin{array}{ll}\mathbf{0}^{T} & 1\end{array}\right]^{T}$. Subsequently, we define the following $(M J L+1) \times(M J L+1)$ matrices $\boldsymbol{K}_{\hat{\boldsymbol{a}}_{j}} \triangleq \hat{\boldsymbol{f}} \hat{\boldsymbol{a}}_{j}^{T}, \boldsymbol{K}_{\hat{\boldsymbol{g}}_{m}} \triangleq \hat{\boldsymbol{f}} \hat{\boldsymbol{g}}_{m}^{T}$, and $\boldsymbol{K}_{\hat{\boldsymbol{b}}_{m}} \triangleq \hat{\boldsymbol{f}} \hat{\boldsymbol{b}}_{m}^{T}$, In addition, we define the symmetric matrices $\boldsymbol{Y} \in \mathbb{R}^{M J L \times M J L}$ and $\boldsymbol{\Omega} \in \mathbb{R}^{(M J L+1) \times(M J L+1)}$ to be $\boldsymbol{Y} \triangleq \boldsymbol{y} \boldsymbol{y}^{T}$ and $\boldsymbol{\Omega}=\hat{\boldsymbol{y}} \hat{\boldsymbol{y}}^{T}$, in particular, $\boldsymbol{\Omega}=\left[\begin{array}{cc}\boldsymbol{Y} & \boldsymbol{y} \\ \boldsymbol{y}^{T} & 1\end{array}\right]$. Using those matrices, the optimization problem in (8) can be cast as

$$
\begin{array}{cl}
\max _{\boldsymbol{\Omega}} \min _{m \in \mathcal{M}} & \frac{\operatorname{Tr}\left(\boldsymbol{K}_{\hat{\boldsymbol{b}}_{m}} \boldsymbol{\Omega}\right)}{\operatorname{Tr}\left(\boldsymbol{K}_{\hat{\boldsymbol{g}}_{m}} \boldsymbol{\Omega}\right)}, \\
\text { subject to } & \frac{1}{2} \operatorname{Tr}\left(\boldsymbol{K}_{\hat{\boldsymbol{a}}_{j}} \boldsymbol{\Omega}\right) \leq 1, \quad \forall j \in \mathcal{J}, \\
& \frac{1}{2} \operatorname{Tr}\left(\boldsymbol{K}_{\hat{\boldsymbol{g}}_{m}} \boldsymbol{\Omega}\right) \leq \frac{1}{\rho} P_{m}^{\max }+P_{m, \mathrm{C}}, \quad \forall m \in \mathcal{M}, \\
& \\
\boldsymbol{\Omega} \succeq \mathbf{0}, & \text { (9b) } \\
\operatorname{diag}(\boldsymbol{\Omega})=1, & \text { (9d) } \\
\operatorname{rank}(\boldsymbol{\Omega})=1 . & \text { (9e) }
\end{array}
$$

This formulation is non-convex due to both the objective function (9a) and the rank-1 constraint (9f). However, noting that after dropping rank-1 constraint, the resulting problem is quasi-convex [10], which can be efficiently tackled using the bisection method. Using a new variable, $\mathcal{E}_{0}$, we reformulate the relaxed problem as

$$
\begin{aligned}
& \max _{\mathcal{E}_{0}, \boldsymbol{\Omega}} \mathcal{E}_{0}, \\
& \text { subject to } \operatorname{Tr}\left(\mathcal{E}_{0}\left(\boldsymbol{K}_{\hat{\boldsymbol{g}}_{m}}-\boldsymbol{K}_{\hat{\boldsymbol{b}}_{m}}\right) \boldsymbol{\Omega}\right) \leq 0, \forall m \in \mathcal{M}, \\
& (9 \mathrm{~b})-(9 \mathrm{e}) .
\end{aligned}
$$

To obtain the maximum value of $\mathcal{E}_{0}$, we solve a series of convex feasibility problems, each of which is of the form

find $\Omega$,

subject to $\operatorname{Tr}\left(\mathcal{E}_{0}\left(\boldsymbol{K}_{\hat{\boldsymbol{g}}_{m}}-\boldsymbol{K}_{\hat{\boldsymbol{b}}_{m}}\right) \boldsymbol{\Omega}\right) \leq 0, \forall m \in \mathcal{M}$,

$$
(9 b)-(9 e)
$$

where $\mathcal{E}_{0}$ is fixed at each iteration of the bisection method and it lies in $\left[0, \mathcal{E}_{\text {max }}\right]$, where $\mathcal{E}_{\text {max }}=\min _{m \in \mathcal{M}}\left(\frac{\boldsymbol{b}_{m}^{T} \mathbf{1}}{P_{m, \mathrm{C}}}\right)$. Let $\Omega^{*}$ represent the optimal solution of (10) corresponding to the optimal value of $\mathcal{E}_{0}$. If $\Omega^{*}$ has a rank one, the relaxation is tight. Otherwise, we use Gaussian randomization [8] in order to obtain an approximate solution. The idea behind of the Gaussian randomization technique 
is to tackle a stochastic version of the deterministic problem in (10). Particular, in this technique, several random samples, $T$, are first generated from a multivariate Gaussian distribution with the covariance matrix of $\Omega^{*}$. Then, the random vector that maximizes the objective in (6) is selected as the sub-optimal solution.

The SDR-based Gaussian randomization technique is summarized in Algorithm 1.

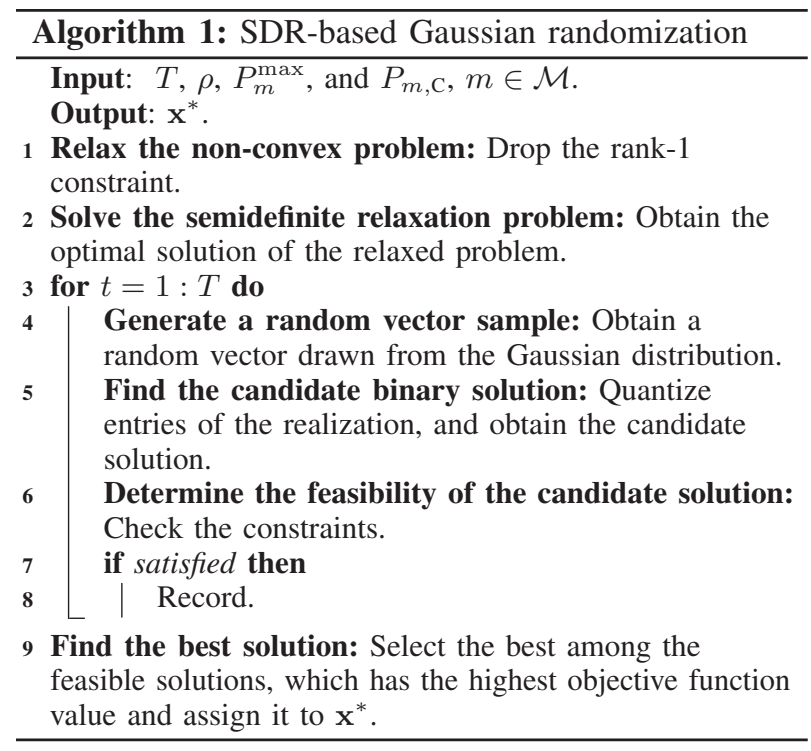

\section{A. Complexity Discussion}

The proposed algorithm uses the bisection method to solve the problem in (10). This method requires $\log _{2}\left(\mathcal{E}_{\max } / \kappa\right)$ number of iterations for the convergence, where $\kappa>0$ is the solution accuracy. Each iteration involves solving a convex PSD-constrained problem, with the computational complexity of $\mathcal{O}\left((M J L)^{3.5}\right)$ [8] Finally, it uses the Gaussian randomization technique. At this step, the complexity of generating $T$ random samples for which the objective is evaluated is $\mathcal{O}\left((M J L)^{2} T\right)$ [8]. Therefore, the total computational complexity can be found as $\mathcal{O}\left((M J L)^{3.5} \log _{2}\left(\mathcal{E}_{\max } / \kappa\right)+(M J L)^{2} T\right)$. Even though the proposed centralized algorithm has polynomial-time complexity, the order of the polynomial complexity is relatively high. Hence, it is applicable in small-to-medium-size networks. For large-size networks, decentralized algorithms can be more desirable in order to ensure reduced complexity and overhead. However, we have the following remarks:

- One of the most effective means of developing optimization-based decentralized designs is to begin with a centralized design and then, using Lagrangian duality, the formulation is decoupled to facilitate the task of each node in the network to optimize its parameters locally. In other words, centralized formulations are usually useful in developing decentralized ones.

- A decentralized formulation can be regarded as a centralized one, but with decoupling constraints. This implies that the solutions generated through the centralized formulation constitute an upper bound on the solutions generated through their decentralized counterparts. In other words, the centralized formulations developed in our work will not only serve as starting points for developing effective decentralized algorithms, but also to serve a benchmark for validating and examining their efficacy.

\section{Simulation Results}

In this section, the EE performance of the proposed resource allocation scheme in an OFDMA network is evaluated with simulations. We assume that the path loss model is expressed as $128.1+37.6 \log _{10}(d)$ for the user-to-BS links [11], where $d$ denotes the distance in kilometers. The log-normal distribution with a standard deviation of $8 \mathrm{~dB}$ is used to model the shadowing factor. The power-level set consists of $L$ equally located points in $\left[0.05 P_{m}^{\max }, 0.5 P_{m}^{\max }\right]$, and $P_{m}^{\max }=P^{\max }, \forall m \in \mathcal{M}$, unless otherwise stated. Furthermore, we assume that noise power spectral density is $-174 \mathrm{dBm} / \mathrm{Hz}$, the bandwidth of each RB is $180 \mathrm{kHz}$, and the power amplifier efficiency is $38 \%$. We use 100 Monte Carlo simulations to obtain the average performance. The optimization problem is solved using the SDPT3 package [12] and the randomization technique is implemented using 1000 independent randomization samples.

Fig. 1 considers a network with 4 RBs, 3 users, 2 power levels. In this network, we assume that the static circuitry power consumption is $25 \mathrm{dBm}$. In Fig. 1, the performance of proposed scheme is compared against the optimal solution derived through exhaustive search. From this figure, it can be readily observed that the performance of the proposed technique is close to the optimal one, especially at high values of the transmit power. For instance, at the transmit power of $31 \mathrm{dBm}$, the gap between the minimum EE and the one generated by the SDR-technique is $2.97 \times 10^{4}$ bits/Joule, which is about $1 \%$ of the objective value obtained by exhaustive search. Moreover, for completeness of presentation, a comparison of these two algorithms in terms of EE fairness is given in Fig. 2. For quantifying the EE fairness among multiple users, we adopt Jain's fairness index [13]. ${ }^{2}$ Since the adopted EE optimization metric, viz., max-min fair EE,

\footnotetext{
${ }^{2}$ Jain's index is widely used as a (numeric) quantitative fairness metric; a higher value of Jain's index corresponds to a fairer rate allocation. Particularly, it is defined as $F(\mathbf{x})=$ $\frac{\left(\sum_{m \in \mathcal{M}} \sum_{j \in \mathcal{J}} \sum_{\ell \in \mathcal{P}} r_{m}^{j \ell} x_{m}^{j \ell}\right)^{2}}{M \sum_{m \in \mathcal{M}}\left(\sum_{j \in \mathcal{J}} \sum_{\ell \in \mathcal{P}} r_{m}^{j \ell} x_{m}^{j \ell}\right)^{2}}$. It is a continuous non-convex function, $F(\mathbf{x}): \mathbb{R}_{m}^{+} \rightarrow \mathbb{R}^{+}$, with values in the interval $\left[\frac{1}{M}, 1\right]$.
} 


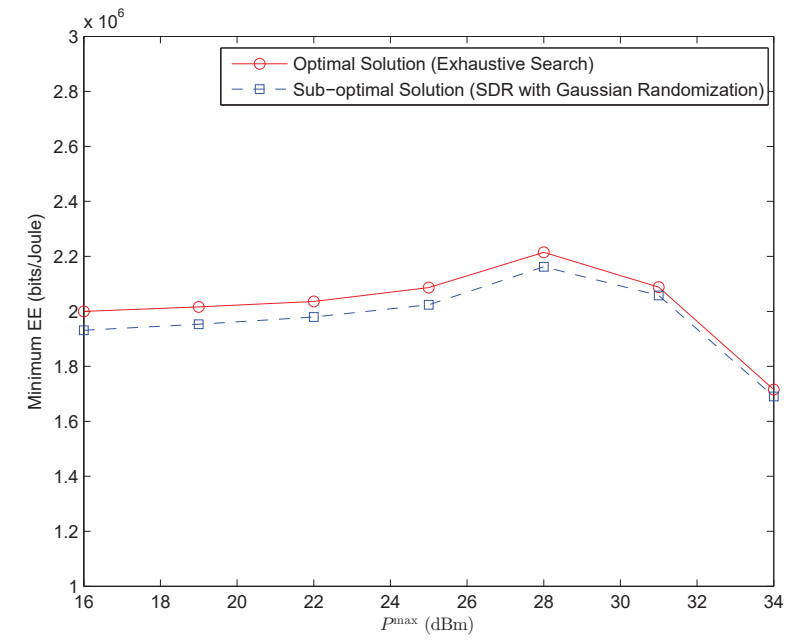

Fig. 1. Performance of the proposed sub-optimal technique against the optimal one.

improves EE fairness between different users as much as possible, as expected, the achieved EE Jain's fairness index is high, specifically, higher than 0.9 for all values of the transmit power.

Fig. 3 considers a network with $10 \mathrm{RBs}, 5$ users, and the different number of power levels, i.e., 2, 4, and 8. In this network, we assume that the static circuitry power consumption is $25 \mathrm{dBm}$. Fig. 2 demonstrates the impact of the number of power levels on the average network performance. One can see that for a specific transmit power, the minimum EE enhances as the number of power level raises. This is due to the fact that the increase of the number of power level provides extra degrees of freedom in the energy-efficient resource allocation optimization. Another important observation is that the impact of the number of power levels on max-min EE optimization becomes less significant as the transmit power increases.

Lastly, Fig. 4 considers a network with 8 RBs, 4 users. In this network, we assume that the static circuitry power consumption is $25 \mathrm{dBm}$, and the number of power levels varies from 1 to 11 . Fig. 4 reveals the average computational time required by the proposed algorithm. From this figure, it can be observed that the proposed algorithm requires a polynomial amount of computational time.

\section{CONCLUSION}

In this work, we addressed the problem of optimizing resource allocation in uplink OFDMA networks for providing the EE fairness among the users while considering discrete power levels. Particularly, we focused on how to maximize the minimum user $\mathrm{EE}$ in the network by jointly optimizing RB and discrete power allocation. We first formulated the optimization problem, but unfortunately, the resulting formulation was not

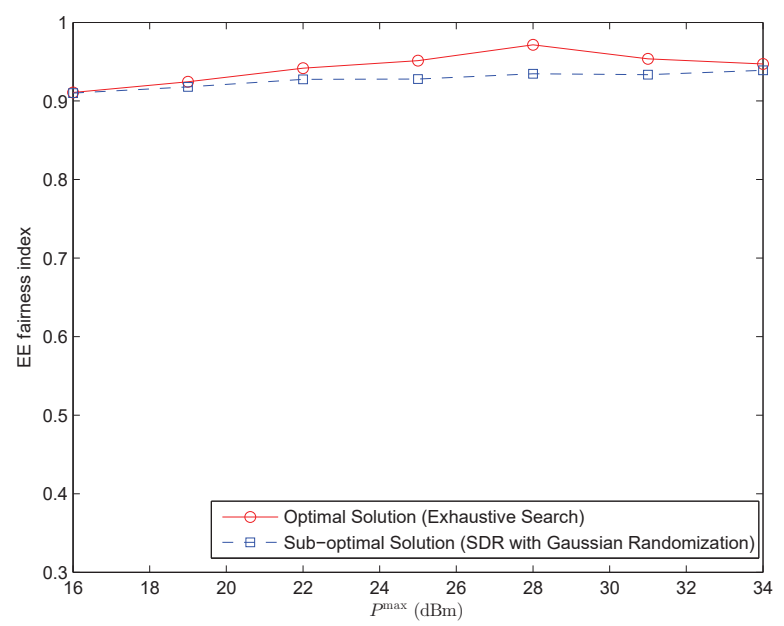

Fig. 2. EE fairness among the users.

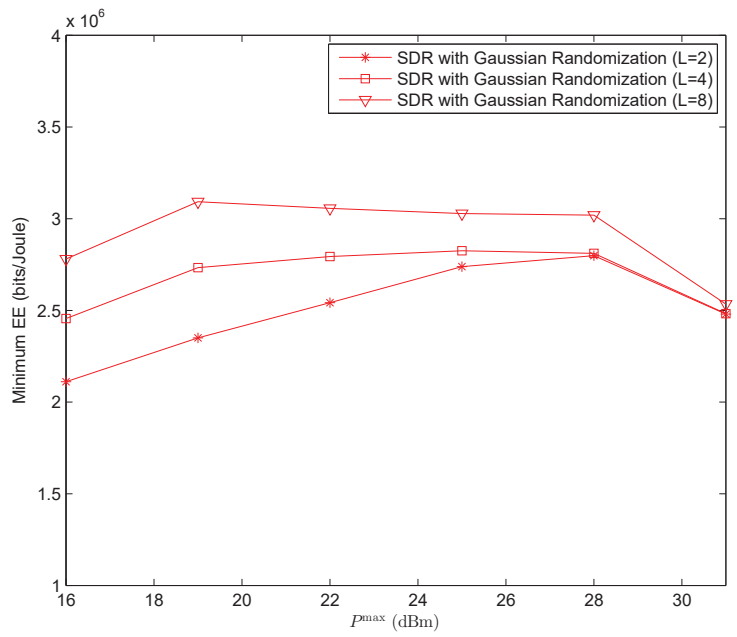

Fig. 3. Performance of the proposed technique with the different number of power levels.

convex. In order to tackle this difficulty, we proposed an SDR-based technique with polynomial time complexity that approximates the solution for the max-min $\mathrm{EE}$ problem using a convex program. The numerical results indicated that the proposed technique does not only achieve the desirable improvement on the users' EE, but also ensures EE fairness among the users. As future works, we plan to put emphasis on distribute solutions, and to investigate the joint optimization of user association, RBs and discrete power allocations for the EE maximization in interference-limited OFDMA-based networks.

\section{REFERENCES}

[1] "Cisco visual networking index: Global mobile data traffic forecast update, 2015-2020," Feb. 2016. [Online]. Available: https: //www.cisco.com/c/en/us/solutions/collateral/service-provider/ visual-networking-index-vni/vni-forecast-qa.pdf 


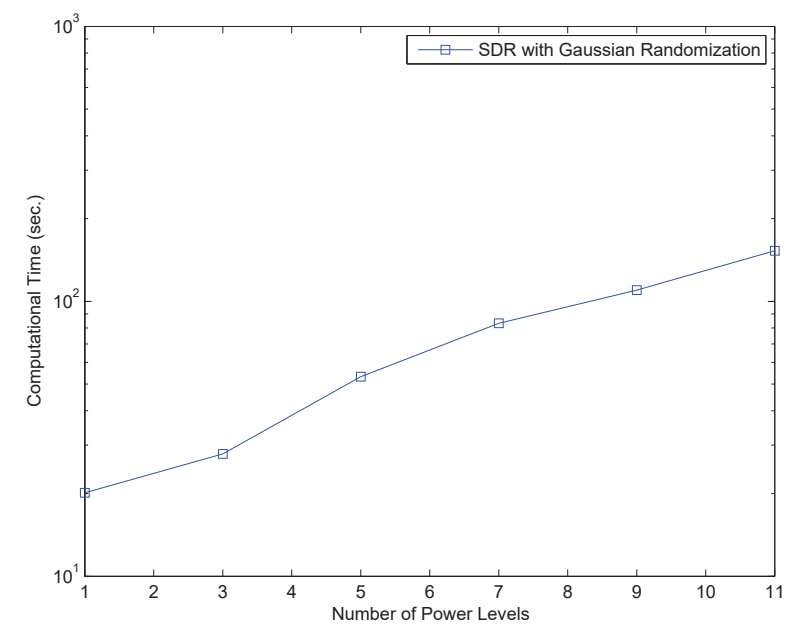

Fig. 4. Computational time versus network size.

[2] D. Feng, C. Jiang, G. Lim, L. J. Cimini, G. Feng, and G. Y. Li, "A survey of energy-efficient wireless communications," IEEE Commun. Surv. Tutorial, vol. 15, no. 1, pp. 167-178, Feb. 2013.

[3] H. Sokun, R. H. Gohary, and H. Yanikomeroglu, "A novel approach for QoS-aware joint user association, resource block and discrete power allocation in hetnets," IEEE Trans. Wireless Commun., vol. 16, no. 11, pp. 7603-7618, Nov. 2017.

[4] Y. Li, M. Sheng, X. Wang, Y. Zhang, and J. Wen, "Max-min energy-efficient power allocation in interference-limited wireless networks," IEEE Trans. Veh. Technol., vol. 64, no. 9, pp. 43214326, Sep. 2015.

[5] C. Guo, B. Liao, L. Huang, X. Lin, and J. Zhang, "On convexity of fairness-aware energy-efficient power allocation in spectrumsharing networks," IEEE Commun. Lett., vol. 20, no. 3, pp. 534537, Mar. 2016.

[6] Y. Li, M. Sheng, C. W. Tan, Y. Zhang, Y. Sun, X. Wang, Y. Shi, and J. Li, "Energy-efficient subcarrier assignment and power allocation in OFDMA systems with max-min fairness guarantees," IEEE Trans. Commun., vol. 63, no. 9, pp. 3183-3195, Sep. 2015.

[7] E. Bedeer, A. Alorainy, M. J. Hossain, O. Amin, and M.-S. Alouini, "Fairness-aware energy-efficient resource allocation for AF cooperative OFDMA networks," IEEE J. Select. Areas Commun., vol. 33, no. 12, pp. 2478-2493, Dec. 2015.

[8] Z.-Q. Luo, W.-K. Ma, A. M.-C. So, Y. Ye, and S. Zhang, "Semidefinite relaxation of quadratic optimization problems," IEEE Signal Process. Mag., vol. 27, no. 3, pp. 20-34, May 2010.

[9] H. Sokun, E. Bedeer, R. Gohary, and H. Yanikomeroglu, "Optimization of discrete power and resource block allocation for achieving maximum energy efficiency in OFDMA networks," IEEE Access, vol. 5, pp. 8648-8658, May 2017.

[10] S. Boyd and L. Vandenberghe, Convex Optimization. Cambridge Univ. Press, 2004.

[11] 3rd Generation Partnership Project (3GPP) TR 36.839, "Evolve universal terrestrial radio access (E-UTRA); Mobility enhancements in heterogeneous networks," v11.1.0, Jan. 2013.

[12] M. Grant and S. Boyd, "CVX: Matlab software for disciplined convex programming, version 2.1," Mar. 2014. [Online]. Available: http://cvxr.com/cvx

[13] R. Jain, D.-M. Chiu, and W. R. Hawe, "A quantitative measure of fairness and discrimination for resource allocation in shared computer system," Digital Equipment Corporation, Tech. Rep. DEC-TR-301, 1984. [Online]. Available: http: //www1.cse.wustl.edu/jain/papers/ftp/fairness.pdf 